\title{
Out of mind, but not out of sight: Intentional control of visual memory
}

\author{
YUKO YOTSUMOTO and ROBERT SEKULER \\ Brandeis University, Waltham, Massachusetts
}

\begin{abstract}
Does visual information enjoy automatic, obligatory entry into memory, or, after such information has been seen, can it still be actively excluded? To characterize the process by which visual information could be excluded from memory, we used Sternberg's $(1966,1975)$ recognition paradigm, measuring visual episodic memory for compound grating stimuli. Because recognition declines as additional study items enter memory, episodic recognition performance provides a sensitive index of memory's contents. Three experiments showed that an item occupying a fixed serial position in a series of study items could be intentionally excluded from memory. In addition, exclusion does not depend on lowlevel information, such as the stimulus's spatial location, orientation, or spatial frequency, and does not depend on the precise timing of irrelevant information, which suggests that the exclusion process is triggered by some event during a trial. The results, interpreted within the framework of a summed similarity model for visual recognition, suggest that exclusion operates after considerable visual processing of the to-be-excluded item.
\end{abstract}

The visual world teems with countless objects and events. To restrict incoming information to a manageable flow, the visual system selectively filters that information, prioritizing on the basis of such attributes as spatial location, color, and direction of motion and on the basis of behavioral relevance. The consequences of such filtering have been well documented in psychophysical and in physiological studies (e.g., Hopfinger, Buonocore, \& Mangun, 2000). An analogue to such visual filtering has been demonstrated in memory research, particularly in studies of intentional forgetting as realized in the directedforgetting paradigm (for a review, see MacLeod, 1998). In a typical directed-forgetting experiment, subjects are instructed to forget some subset of the items that they have studied. Carrying out this instruction improves recall of the remaining items, relative to conditions in which all the studied items must be remembered. In addition to this benefit from directed forgetting, some studies have revealed a cost: The presence of items that directed forgetting has rendered task irrelevant can impair recall of task-relevant items. Although directed forgetting has been well documented for verbal materials, evidence is scant that such intentional filtering can alter the operation of nonverbal memory. However, costs associated with task-

This research was supported by Air Force Office of Scientific Research Grant F49620-03-1-0376 and National Institutes of Health Grant MH55687. We thank Michael J. Kahana and Allison B. Sekuler for many useful suggestions. Correspondence concerning this article may be addressed to R. Sekuler, Volen Center for Complex Systems, Brandeis University, Waltham, MA 02454 (e-mail: vision@brandeis.edu).

Note-This article was accepted by the previous editorial team, when Colin M. MacLeod was Editor. irrelevant items have been demonstrated in auditory masking tasks and in visuospatial memory tasks (Crowder, 1978; Parmentier, Tremblay, \& Jones, 2004). As for visual memory, under some circumstances, behaviorally irrelevant visual inputs seem to enjoy automatic, obligatory entry into visual memory, which allows behaviorally irrelevant visual material to interfere with retrieval for taskrelevant information. Such interference has been seen in visual memory for various spatial attributes, such as the distance separating dots (Hole, 1996) and the locations of cells in two-dimensional matrices (Toms, Morris, \& Foley, 1994; Washburn \& Astur, 1998). However, other studies suggest that interference is not inevitable but depends on the stimulus dimension and task details (e.g., Lalonde \& Chaudhuri, 2002; Ostendorf, Finke, \& Ploner, 2004).

For a highly sensitive assay of voluntary, active control over items' entry into visual memory, we adapted Sternberg's $(1966,1975)$ recognition memory paradigm, examining episodic recognition for series of compound gratings. Previously, Kahana and Sekuler (2002) showed that such stimuli afford many advantages when used as probes of memory and in computational modeling. Because the set of memoranda varies from one trial to the next, successful recognition performance on any trial requires access to episodic information - that is, information about the items seen on that trial. Systematic variation of perceptual differences among stimuli produces highly predictable changes in recognition performance (Kahana, Zhou, Geller, \& Sekuler, in press; Sekuler, Kahana, McLaughlin, Golomb, \& Wingfield, 2005; Zhou, Kahana, \& Sekuler, 2004). These changes, in turn, support detailed quantitative accounts of recognition memory and intentional ignoring. Also, we propose that in comparison with such stimuli as nameable words or pictures of com- 
mon objects, compound gratings presented briefly and in rapid succession resist consistent verbal description and rehearsal. For one thing, as can be seen from the examples in Figure 1, compound gratings' perceptual homogeneity and confusability make it difficult to assign consistent verbal labels to such stimuli, particularly when they are presented briefly and in rapid succession. In addition, when subjects are tested with compound gratings, rather than with words or pictures, their encephalographic records show very much weaker oscillatory correlates of subvocal rehearsal (Hwang et al., 2005). Finally, recognition tests with compound gratings show no primacy effect, such as might be expected had the memoranda been verbally labeled and rehearsed (Kahana \& Sekuler, 2002).

Our experiments exploited the fact that episodic recognition memory for compound grating stimuli declines as additional items enter into memory and that the decline depends on the perceptual relationships among the items in memory (Kahana \& Sekuler, 2002; Kahana et al., in press). Because of memory's dependence on the number of study items, recognition performance provides a sensitive index of memory's contents. If subjects could voluntarily completely exclude from memory one study item, recognition with $n+1$ items would be comparable to recognition achieved with $n$ items. If exclusion were only partially successful, recognition would fall somewhere between that expected for $n$ and for $n+1$ items. Such comparisons provide the foundation of our analysis.

The basic manipulation in one condition of our experiments encouraged subjects to view but filter out or ignore a stimulus presented in one particular serial position. In designing our experiments, we were aware that instructions such as ours can backfire, producing an effect opposite to what was intended. Such ironic effects have been well studied by Wegner (1994) and include memoryrelated effects (Macrae, Bodenhausen, Milne, \& Ford, 1997; Wenzlaff \& Wegner, 2000). If our instructions to ignore generated an ironic effect, our manipulation might generate heightened memory for the to-be-ignored stimulus.

\section{EXPERIMENT 1}

This experiment determined whether subjects could intentionally exclude from memory one of a series of study items. On each trial, the subjects first saw either one or two briefly presented compound gratings, which made up the study series for that trial. The study series was followed by a probe stimulus - hereafter, $p$ - a compound grating that either replicated one of the study stimuli or, with equal probability, was novel for that trial. The subject made a yes-no recognition response, judging whether $p$ replicated a study item. We measured recognition under three experimental conditions, including one in which the subjects attempted to ignore the study item in the second serial position of each two-item study series. Performance in this condition was compared with performance when either both items or just one had to be remembered.

To maximize the likelihood of demonstrating effective ignoring, different conditions were presented in blocks of trials, during which the subjects attempted exclusion or not. Also, study stimuli that were to be ignored differed in orientation by $45^{\circ}$ from other stimuli, and to-be-ignored items always occurred at a fixed time within a trial. These conditions were meant to facilitate intentional ignoring.

\section{Method}

Subjects. The subjects were 8 paid volunteers whose ages ranged from 18 to 21 years. They had normal or corrected-to-normal visual acuity, as measured with Snellen targets, and normal contrast sensitivity, as measured with Pelli-Robson charts (Pelli, Robson, $\&$ Wilkins, 1988). The subjects were naive as to the experiment's purpose.

Stimuli. The stimuli were compound gratings, each synthesized by adding two orthogonal sinusoidal components of equal contrast. The stimuli were generated and presented using MATLAB and extensions from the Psychophysics and Video Toolboxes (Brainard, 1997; Pelli, 1997). Gratings were presented in either of two orientations, upright or oblique. Upright gratings were synthesized by summing one vertical and one horizontal sinusoid, generating a luminance profile,

$$
L_{x, y}=L_{\text {avg }}\left[1+0.2\left(\frac{\cos 2 \pi f(x)+\cos 2 \pi g(y)}{2}\right)\right],
$$

where $L_{\text {avg }}$ is mean luminance, $f$ is the spatial frequency of the vertical component in cycles per degree, and $g$ is the frequency of the horizontal component. Oblique gratings were generated by applying to Equation 1 a rotation matrix,

$$
L\left(x_{\mathrm{o}}, y_{\mathrm{o}}\right)=\left[\begin{array}{cc}
\cos \theta & \sin \theta \\
-\sin \theta & \cos \theta
\end{array}\right] L\left(x_{\mathrm{u}}, y_{\mathrm{u}}\right),
$$

where the subscripts o and $\mathrm{u}$ signify oblique and upright, respectively. For our experiments, $\theta=45^{\circ}$.

Each stimulus comprised two sinusoidal components in orthogonal orientations. One orientation component was made critical to the subjects' recognition judgments, whereas the other was irrelevant. The critical orientation component was the only component whose spatial frequency could vary among the stimuli on any trial. As a result of its variation, correct recognition judgments depended on memory for this critical orientation component, whose spatial frequency covered the range of $0.5-3$ cycles/degree of visual angle. This variation eliminated any potential benefit from learning to recognize or ignore some particular spatial frequency. The critical orientation was either vertical, in which case we will designate the compound grating as upright, or $45^{\circ}$, in which case we will designate the compound grating as oblique. Irrelevant components were generated by adding a sinusoid orthogonal in orientation to the critical component: a horizontal sinusoidal component for each upright grating and a $135^{\circ}$ sinusoid for each oblique grating. The spatial frequency of an irrelevant component was chosen by a random selection from a uniform distribution spanning 0.5-3 cycles/degree of visual angle. This selection was constrained so that the same frequency was used for all the stimuli in any one trial. For terminological convenience, throughout the rest of this article, we will refer to the critical component's spatial frequency as the stimulus's spatial frequency, without consideration of the irrelevant orientation.

Each compound grating subtended $5^{\circ}$ of visual angle in diameter and was windowed with a bivariate Gaussian function. Stimulus contrast was 0.2 , which was well above the detection threshold but, with a steady veiling luminance on the screen, produced minimal or no visible afterimage. To undermine the usefulness of possible comparisons between corresponding locations on successive gratings, both of the stimulus's orientation components were randomly phase shifted by $0-0.25$ cycle.

To reduce individual differences in recognition performance, we adjusted the stimuli with which each subject would be tested, tailoring differences between stimuli to each subject's own Weber fraction 

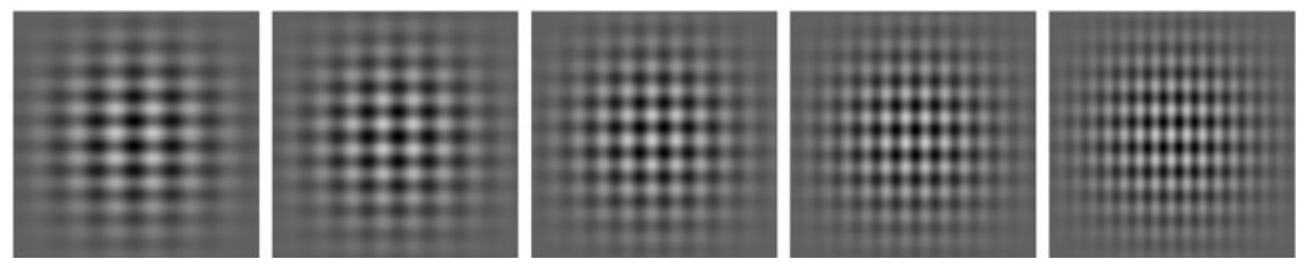

Figure 1. Samples of compound grating stimuli used in all three experiments. The vertical components of the stimuli at the extreme left and right differ in spatial frequency from the center stimulus by \pm 2 Weber fraction units; for the remaining stimuli, the vertical spatial frequency differs from that of the center stimulus by \pm 1 Weber fraction unit. The samples shown here are for upright stimuli; the corresponding oblique stimuli could be produced by rotating these samples about their center by $45^{\circ}$. For ease of visibility, all the stimuli are shown here at higher contrast than that used in the experiments. Note that the irrelevant, horizontal spatial frequency is constant within a trial, as is the irrelevant, $135^{\circ}$ component for $s_{2}$ and $p_{2}$ in the Rem2 condition.

for spatial frequency (Zhou et al., 2004). We used the method of constant stimuli to measure each subject's Weber fraction, presenting a pair of gratings successively on each trial. From one trial to the next, the first grating's vertical spatial frequency varied from 0.5 to 3.0 cycles/deg, whereas the vertical spatial frequency of the second stimulus was set to a fractional multiple of the standard. The subjects made yes-no judgments of whether the two stimuli did or did not match one another and received feedback after each response. To mimic the conditions that would be used in our recognition experiments, we added a horizontal sinusoidal component to the gratings. The frequency of the horizontal component was drawn from the same range as the vertical component's frequency, but because it was kept constant for both gratings on each trial, the horizontal component was rendered irrelevant to the yes-no judgment. With these stimuli and the same timing parameters as those that would later be used in the equivalent experimental condition, we estimated the frequency difference between standard and comparison gratings that produced a mean percent correct of $75 \%$. Across subjects, the resulting Weber fractions ranged from $24 \%$ to $39 \%$.

Apparatus. The stimuli were presented on a 15 -in. computer monitor with a refresh rate of $95 \mathrm{~Hz}$ and a resolution of $800 \times$ 600 pixels. Routines from the Video Toolbox calibrated and linearized the display luminance. Mean screen luminance was maintained at $30 \mathrm{~cd} / \mathrm{m}^{2}$; because all the stimuli had the same mean luminance as this steady background, a stimulus' onset or offset produced no overall luminance transient. The subjects' heads were stabilized with a head- and chinrest; viewing was binocular from a distance of $114 \mathrm{~cm}$. To promote consistent, central viewing, a small, black fixation cross appeared in the center of the display at the beginning of each trial. The subjects were instructed to fixate this cross, which remained on the screen until $200 \mathrm{msec}$ prior to the onset of the trial's first study stimulus. In all the experiments, each compound grating stimulus, study items as well as probes, was presented for $700 \mathrm{msec}$.

Procedure. The subjects were tested in three conditions. Two of them we will designate as Rem 2 and Rem 1 , signifying, respectively, that on each trial two study items or just one were presented and had to be remembered. In the third condition, Ignore, the subjects viewed two study items on each trial but had to remember only the first one; they could ignore the second study item. Figure 2 schematizes the stimulus sequences for each of the three conditions. The following paragraphs will describe each condition in more detail.

Rem 2 condition. On each trial, two study items, $s_{1}$ and $s_{2}$, were presented in succession with an interstimulus interval (ISI) of $1,000 \mathrm{msec}$. After a 1,000-msec delay, they were followed by a probe $\left(p_{1}\right)$, which the observers judged as matching or not matching $s_{1}$. Then, 1,000 msec after the subject's response, a second probe, $p_{2}$, was shown, and the subjects judged whether or not $p_{2}$ matched $s_{2}$. By requiring separate judgments with respect to both $s_{1}$ and $s_{2}$, we were encouraging the subjects to encode and remember both study stimuli. To help the subjects keep the two judgments separate, $s_{1}$ and $p_{1}$ shared the same orientation, $45^{\circ}$ from the orientation shared by $s_{2}$ and $p_{2}$. For one group of subjects, $s_{1}$ and $p_{1}$ were upright, whereas $s_{2}$ and $p_{2}$ were oblique (the upright group); for the other group, $s_{1}$ and $p_{1}$ were oblique, whereas $s_{2}$ and $p_{2}$ were upright (the oblique group).

Reml condition. On each trial, just a single study stimulus $\left(s_{1}\right)$ was presented. This was followed $2,700 \mathrm{msec}$ later by a single probe $\left(p_{1}\right)$. The observers judged whether $p_{1}$ was the same as $s_{1}$ or not. The same orientation was shared by $s_{1}$ and $p_{1}$, which was upright for half the subjects and oblique for the others. Note that the 2,700-msec delay between $s_{1}$ and $p_{1}$ matched the delay in the Rem 2 condition between $s_{1}$ and $p_{1}$, which facilitated comparisons between these two conditions. For one group of subjects, $s_{1}$ and $p_{1}$ were upright; for the other group, $s_{1}$ and $p_{1}$ were oblique.

Ignore condition. On each trial, $s_{1}$ and $s_{2}$ were followed by a single probe, $p_{1}$. A 1,000-msec ISI separated the items, as it did in the Rem 2 condition. The subjects were instructed to view both study items but to ignore $s_{2}$, judging only whether $p_{1}$ matched the first study stimulus, $s_{1}$, or not. To help the subjects perform the task, the orientations of $s_{1}$ and $s_{2}$ always differed by $45^{\circ}$. For one group of subjects, $s_{1}$ and $p_{1}$ were upright, but $s_{2}$ was oblique (the upright group), and for the other group, $s_{1}$ and $p_{1}$ were oblique, but $s_{2}$ was upright (the oblique group).

Previous work showed that recognition performance for stimuli such as ours is strongly influenced by the similarity of probe and study items (Kahana \& Sekuler, 2002). Therefore, for all the conditions, $p_{1}$ 's spatial frequency could differ from that of $s_{1}$ by any of several different, fixed amounts: On half the trials in all the conditions, $p_{1}$ was the same as $s_{1}$; that is, $p_{1}$ differed from $s_{1}$ by zero Weber fraction units. On the remaining trials, $p_{1}$ was equally likely to differ from $s_{1}$ by $\pm 1, \pm 2$, or \pm 3 Weber fraction units. The same constraints were applied to $p_{2}$ and $s_{2}$ in the Rem 2 condition, the only condition in which there was a second probe. In addition, we forced $s_{1}$ and $s_{2}$ to differ always by \pm 2 Weber fraction units, which minimized confusions between those stimuli (Kahana \& Sekuler, 2002).

In a block-randomized design, for each condition, every subject was tested in a session with three separate blocks of trials. The first 10 trials of each 110-trial block were eliminated from data analysis. Three sessions were conducted per subject, producing a total of 300 trials per condition for each subject. Distinctive tones immediately after a response provided feedback about response correctness.

\section{Results and Discussion}

Figure 3 shows the mean proportion of correct responses for each condition. Since differences between vertical and 


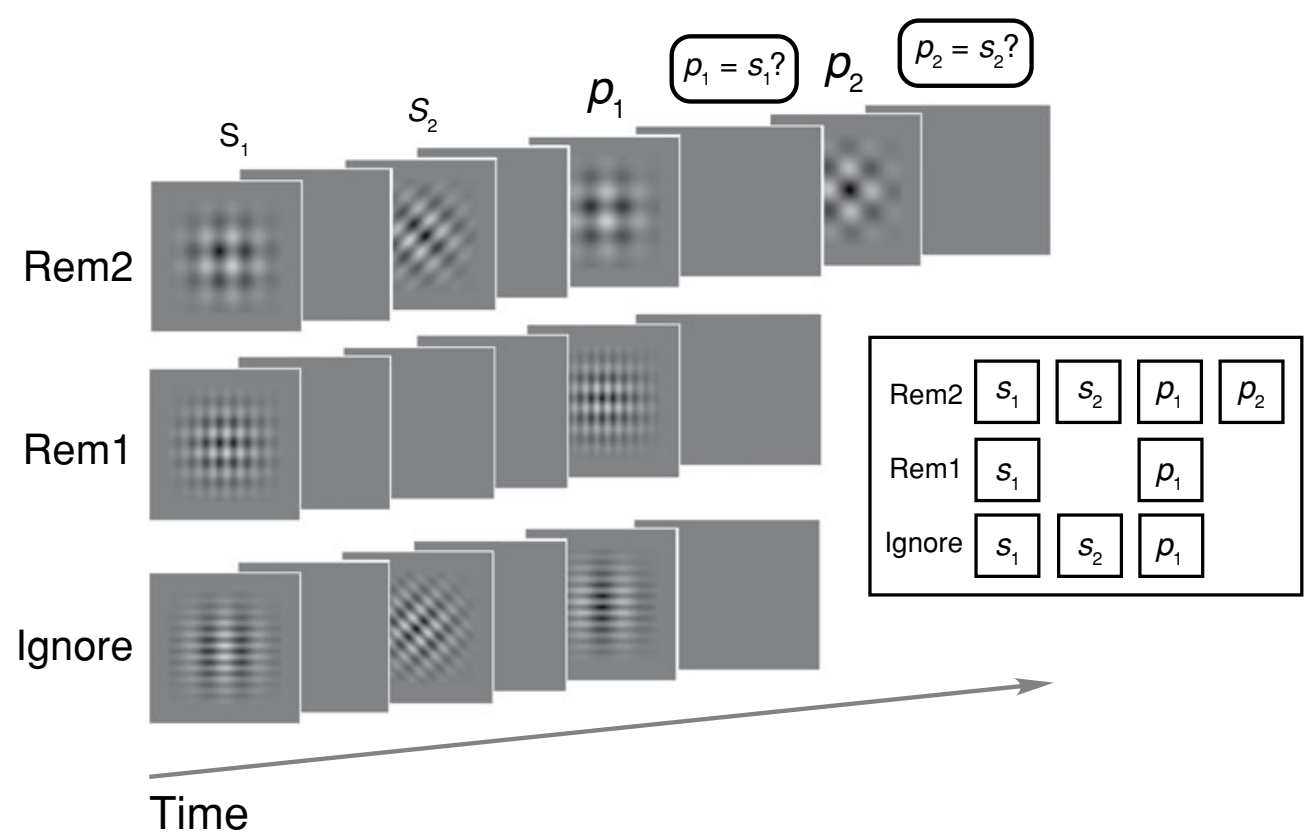

Figure 2. Examples of trial structure and tasks for three conditions. These are examples of stimuli for the upright group: $s_{1}$ and $p_{1}$ have an upright orientation, but $s_{2}$ and $p_{2}$ are oriented obliquely. For the oblique group, vertical stimuli and oblique stimuli were reversed: $s_{1}$ and $p_{1}$ are both oriented obliquely, but $s_{2}$ and $p_{2}$ have an upright orientation. The labels above the stimuli and the questions shown for each condition were not actually present in the stimulus displays; they are given here only as reminders of the judgments that the subjects had to make. The diagrams in the insert summarize the serial order of the stimuli in each of the three conditions.

oblique groups were inconsequential $[F(1,6)=0.182, p>$ $.60]$, the results from the two groups have been combined in the subsequent analyses. In Figure 3, the three gray bars represent mean recognition performance for $p_{1}$ in each of the three conditions; the striped bar shows performance for $p_{2}$ in the Rem 2 condition. Recognition performance differed significantly among conditions $[F(3,18)=27.3$, $p<.001]$. A priori comparisons showed that recognition in the Ignore and the Rem1 conditions did not differ from one another $(p>.40)$, whereas recognition in the Rem2 condition was significantly worse than that for the other two conditions $(p<.01)$. Finally, in the Rem 2 condition, $p_{2}$ was recognized less well than $p_{1}(.01<p<.02)$.

The similarity of the mean recognition scores for the Rem1 and the Ignore conditions suggests that the behaviorally irrelevant stimulus, $s_{2}$, was successfully kept out of memory in the Ignore condition. However, previous work showed that the proportion of correct recognition responses depends strongly on the spatial frequency difference between the probe and the study items (Kahana \& Sekuler, 2002; Zhou et al., 2004). So, for a more sensitive index of differences in memory among experimental conditions, we broke down Figure 3's results according to the spatial frequency difference between the study and the probe items. The outcome of this analysis is shown in Figure 4.

When different $p_{1}-s_{1}$ or $p_{2}-s_{2}$ similarities were taken into account, the proportion of yes responses varied not only across conditions $[F(3,21)=38.4, p<.001]$, but also with the $p-s$ similarities $[F(3,21)=92.9, p<.001]$. In particular, a priori comparisons using the Bonferroni adjustment showed significant differences between the Rem 1 and the Ignore conditions $(p<.01)$ and also between the Ignore and the $\operatorname{Rem} 2 p_{1}(p<.01)$. There was also a significant interaction between conditions and the

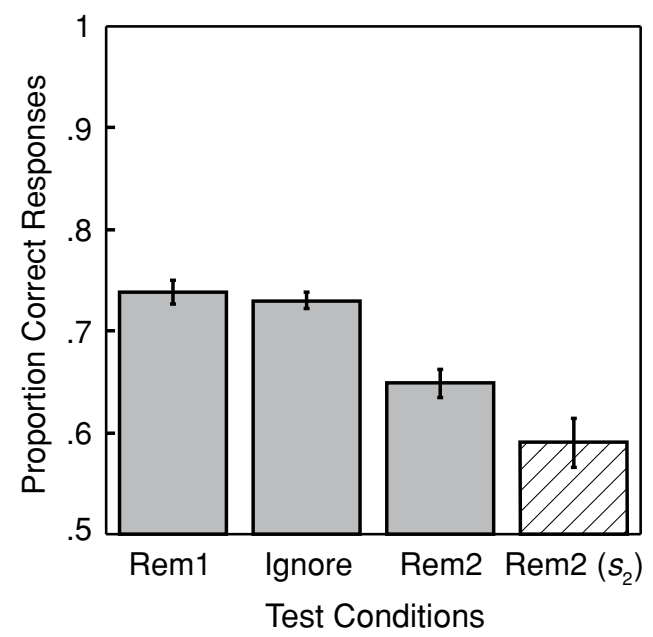

Figure 3. Proportion of correct responses for $p_{1}$ in each condition (gray bars) and for $p_{2}$ in the Rem2 condition (striped bar). Error bars represent standard errors calculated for a withinsubjects, repeated measures design (Loftus \& Masson, 1994). 


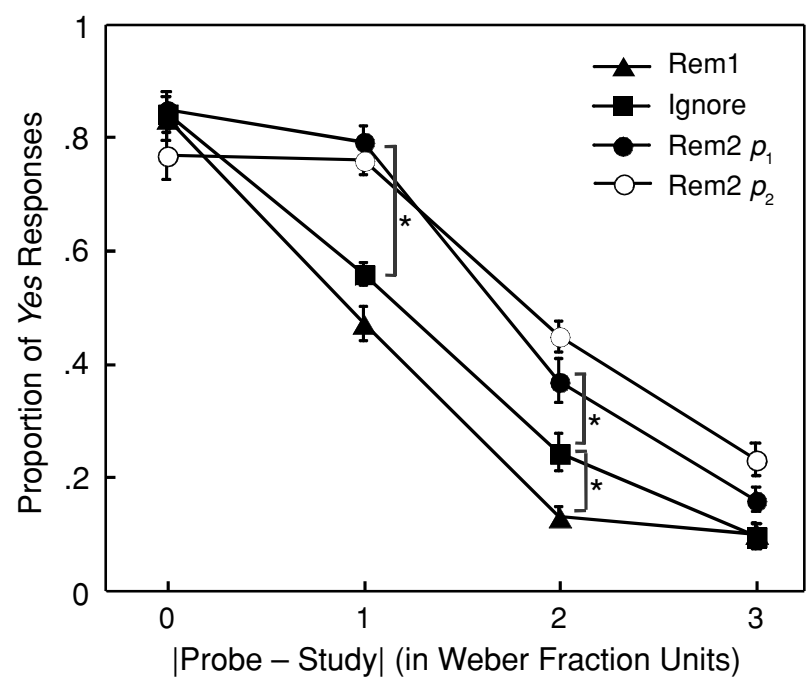

Figure 4. Proportions of yes responses as a function of the spatial frequency difference between $s_{1}$ and $p_{1}$ or $s_{2}$ and $p_{2}$. Values on the $\boldsymbol{x}$-axis are absolute values of the difference, in Weber fraction units. The figure shows the proportions of yes responses in the Rem 1 condition with filled triangles, and those in the Ignore condition with filled squares; the proportions correct for $p_{1}$ in the Rem 2 condition are represented by filled circles, and the corresponding values for $p_{2}$ in that same condition are represented by open circles. Significant differences $(p<.05)$ within each Weber fraction unit are indicated by asterisks. Error bars represent within-subjects standard errors.

$p-s$ spatial frequency differences $[F(9,63)=6.5, p<$ $.001]$. When the $p$ and the $s$ items differed either by three Weber fraction units or by none, the conditions did not differ among themselves after Bonferroni correction. When $s$ and $p$ differed by just one Weber unit, the Ignore condition produced a significantly lower proportion of yes responses for $p_{1}$ than did the Rem2 condition ( $p<$ $.001)$. The corresponding difference between the Ignore and the Rem 1 conditions just failed to reach statistical significance $(.05<p<.06)$. When the spatial frequency difference between the study stimulus and the probe was two Weber units, the Ignore condition was significantly different from both the Rem1 condition $(p<.05)$ and $p_{1}$ in the Rem 2 condition $(p<.05)$. Significant differences within each $p-s$ Weber unit distance are shown with asterisks in Figure 4.

Our results show that the subjects had considerable success in excluding from memory an irrelevant stimulus that had been interposed between a study item and the probe. As Figure 4 demonstrates, this success varied with the spatial frequency difference between $p$ and the matching study item. In particular, when $p$ was not identical to, but also not greatly different from, the study item, performance was degraded by the interposition of a behaviorally irrelevant stimulus, $s_{2}$. Note that trialwise variation in the spatial frequency of the memoranda means that any ignoring observed could not have resulted from low-level learning to ignore a specific spatial frequency or narrow range of spatial frequencies.
Finally, note that recognition of $p_{2}$ in the Rem 2 condition was slightly poorer than the recognition of $p_{1}$ in that same condition. Because $p_{2}$ 's presentation was delayed until the subject made a judgment for $p_{1}$, the difference in performance for the two probes might have reflected a time-dependent degradation in memory for $s_{2}$. On average, the interval between $p_{2}$ and $s_{2}$ was only $\approx 0.75 \mathrm{sec}$ longer than the interval between $p_{1}$ and $s_{1}$. We suspected that this slight increase in delay could not explain the loss in recognition of compound gratings, which is essentially invariant with delays up to $\approx 10$ times as long (Kahana \& Sekuler, 2002; Magnussen, Greenlee, Asplund, \& Dyrnes, 1991; Magnussen, Greenlee, \& Thomas, 1996). However, we decided to assess directly the possible contribution of preprobe delay. Trials were sorted into five equal-width bins, based on the interval between $p_{2}$ and $s_{2}$. A repeated measures ANOVA showed no effect of the interval between $s_{2}$ and $p_{2}$ on the recognition performance for $p_{2}$ $[F(4,28)=1.36, p>.25]$. This suggests that the small but significant difference between recognition $p_{1}$ and $p_{2}$ was not the consequence of a delay in testing $p_{2}$.

This experiment produced strong evidence that $s_{2}$ could be considerably excluded from memory. Before considering the origin of this effect, we had to examine the possibility that the subjects had exploited a simple orienting response in order to exclude $s_{2}$. In particular, we wondered if the subjects might have closed their eyes or averted their gaze around the time of $s_{2}$ 's presentation. To test this idea, we made a video of 3 subjects' eyes during the time they were being tested in the Ignore condition. A light-emitting diode (LED) was mounted on the forehead support, just out of the subjects' field of view. During the presentation of $s_{2}$, the LED was illuminated. A Webcam generated a video image in which the LED and the subjects' eyes were clearly visible. The video was saved to disk for analysis offline. Later, three naive volunteers viewed the videos in order to judge whether the subjects had been looking directly at the computer display during the periods of LED illumination.

All three judges expressed confidence that the subjects had been looking at the display during nearly all of the trials - on average, $97.8 \%$. This result suggests that $s_{2}$ 's voluntary, substantial exclusion from memory probably did not result from the subjects' shifting their gaze to avoid viewing $s_{2}$. We followed up by calibrating the validity of these judgments against a new, staged video in which we controlled whether the subjects were or were not looking at the monitor. With this calibration video, judges correctly distinguished the gaze position shown in the video some $95.5 \%$ of the time. This supports the idea that during the experiment, the subjects had not averted their gaze or closed their eyes in order to exclude $s_{2}$.

\section{EXPERIMENT 2}

\section{Purpose}

In Experiment 1, the behaviorally irrelevant stimulus, $s_{2}$, differed in orientation from $s_{1}$ and $p_{1}$. This orienta- 
tion difference was meant to aid the subjects' efforts to exclude the irrelevant stimulus. But is such a difference necessary for memory exclusion? The answer is important theoretically because it could offer insight into the process or processes that control ignoring. If intentional ignoring persisted in the absence of orientation differences, it would suggest that exclusion does not depend on low-level spatial differences, of which orientation is one example. Specifically, with an orientation difference between the to-be-ignored item and the other list items, the subjects could perhaps filter out all occurrences of the irrelevant item's orientation throughout an entire block of trials, whereas if the orientation were held constant and shared by all list items, the subjects would be deprived of that potential cue. We therefore repeated the Ignore condition, with the orientation difference eliminated.

\section{Method}

The stimuli and procedures were the same as those in Experiment 1 's Ignore condition, except that $s_{1}, s_{2}$, and $p_{1}$ all shared the same orientation. The 8 subjects from Experiment 1 served in 310 trials, of which the first 10 were treated as practice. Four subjects made up the oblique group, and 4, the upright group. The subjects in the upright group were shown only upright gratings; the subjects in the oblique group were shown only oblique gratings.

\section{Results and Discussion}

Since the oblique and the upright groups' results were not noticeably different $(p>.50)$, we ignored group membership in all the analyses. Figure 5A shows the proportion of yes responses as a function of spatial frequency differences between the study stimulus, $s_{1}$, and the probe, $p_{1}$. For comparison, the range bracketed by the standard errors in
Experiment 1's Ignore condition is shown as a gray band; in addition, the proportion of yes responses from Experiment 1's Rem 2 condition is shown as open circles. An ANOVA confirmed what can be seen in Figure 5A-namely, that the proportion of yes recognition responses in Experiments 1 and 2 did not differ $[F(1,7)=0.901, p=.37]$. This experiment tested only the Ignore condition, which was then compared with the results from Experiment 1, with the same subjects. Such comparisons between experiments must be made with caution because, with shared testing conditions, the two sets of results could be differentially influenced by the operation of any practice effect. However, a pilot study showed no change in ignoring even with extensive practice, which strengthens our belief that the results from the two experiments can, in fact, be compared.

Numerous psychophysical and physiological studies have shown that attention can be allocated to some specific low-level feature, either diminishing or enhancing that feature (e.g., Carrasco, Ling, \& Read, 2004; Luck, Chelazzi, Hillyard, \& Desimone, 1997; Slotnick, Schwarzbach, \& Yantis, 2003; Somers, Dale, Seiffert, \& Tootell, 1999; Treue, 2001; Watanabe et al., 1998). If the processing and storage of visual information for memory had been dependent on orientation, a low-level stimulus characteristic, Experiments 1 and 2 would have yielded differential success in exclusion from memory. Because the behaviorally irrelevant items in Experiment 1 differed in orientation from other items on each trial, orientation-dependent processing and storage could have aided exclusion in that experiment. However, we found no difference between the Ignore conditions in Experiment 1 and in Experiment 2, which supports the idea that the success of intentional ig-
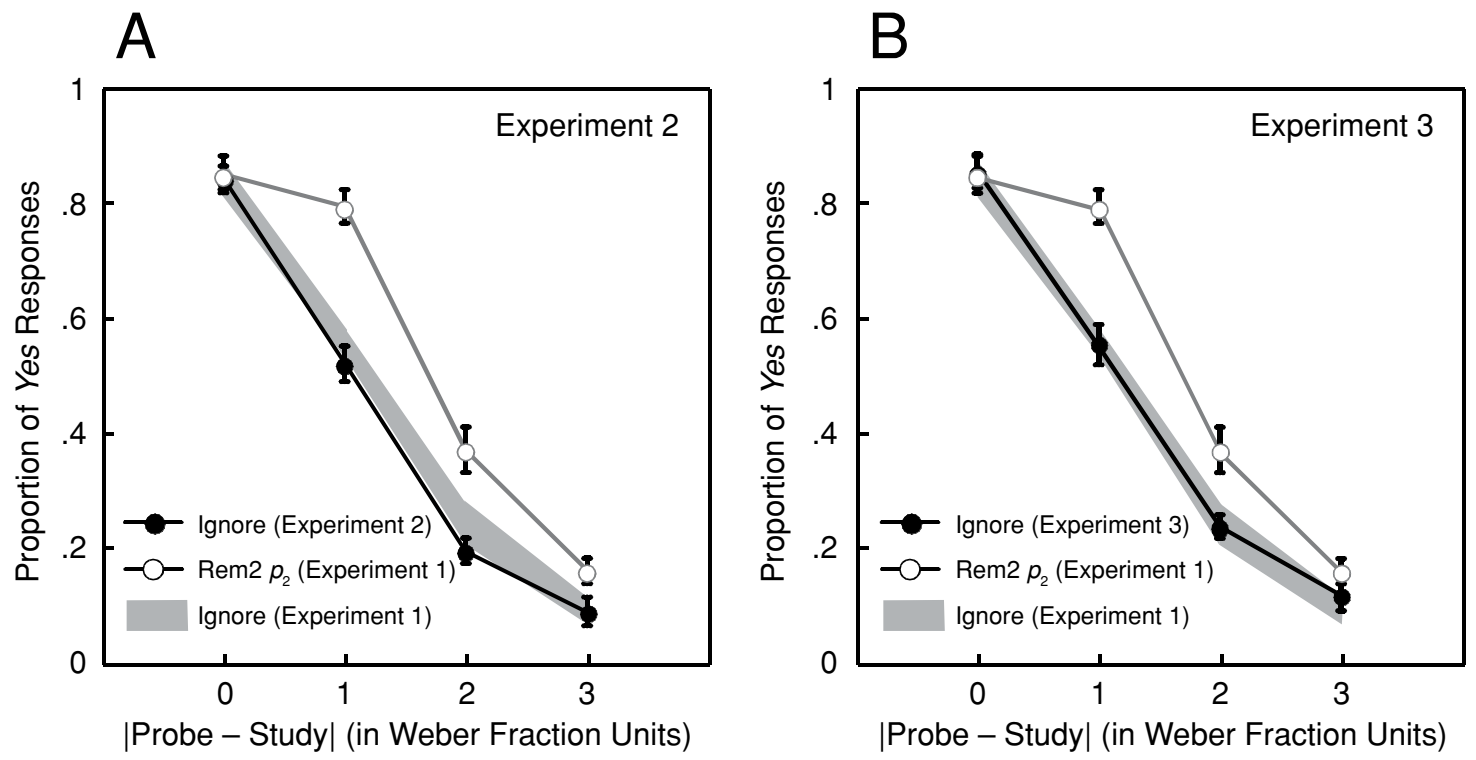

Figure 5. Proportions of yes responses as a function of the absolute value of the difference in spatial frequency between the study stimulus and the probe, in Weber fraction units. (A) In Experiment 2, all the stimuli shared the same orientation. Within-subjects standard errors are shown by the vertical bars around each data point. (B) In Experiment 3, the timing of $s_{2}$ was unpredictable. To facilitate comparison with the results from Experiment 1, in both panels, the range bracketed by standard errors in Experiment 1's Ignore condition is shown as a gray region; in addition, in each panel, the open circles represent the results from Experiment 1's Rem2 condition. 
noring does not depend crucially on low-level differences between stimuli.

\section{EXPERIMENT 3}

Experiment 1 showed that subjects could, at least partially, exclude from memory a behaviorally irrelevant stimulus. Experiment 2 showed that this effect did not require an orientation difference between relevant and irrelevant stimuli but left open the question of what actually triggers the process of exclusion. One possibility emphasizes the timing of the irrelevant stimulus. In both experiments, the ISI and stimulus durations were constant from trial to trial, with $s_{2}$ always displayed exactly $1,700 \mathrm{msec}$ after the trials started. As a result, the subjects could have learned to exclude visual input during a particular interval within a trial. This would resemble a recently demonstrated, timedependent neural inhibition of motion processing (Bisley, Zaksas, Droll, \& Pasternak, 2004). Alternatively, in our experiments, ignoring might have been triggered by some event-based process, by registering the offset of $s_{1}$ and/or the onset of $s_{2}$. Such processes would resemble the active gating mechanisms that filter out irrelevant or extraneous events that occur on trials in psychophysical and physiological studies of sensory processing. These extraneous events occur on every trial-before, during, or after a trial's critical visual stimulus (see, e.g., Seidemann, Zohary, $\&$ Newsome, 1998). In order to distinguish between timebased and event-based accounts, we varied the ISI and the preprobe delay, which made it difficult for the subjects to initiate exclusion until they actually saw the onset of $s_{2}$.

\section{Method \\ The stimuli and procedures were the same as those in Experi- ment 1 's Ignore condition, except that the ISI and the preprobe delay varied randomly. The pair of values for each trial's ISI and preprobe delay were drawn independently from the same uniform random distribution, which spanned a range of 300-1,700 msec. Taking into account the duration ( $700 \mathrm{msec}$ ) of $s_{2}$, these random delays caused the onset of $p_{1}$ to vary from 1,300 to $4,100 \mathrm{msec}$. The subjects were the 8 individuals who had participated in the preceding experiments. Again, half the subjects made up the upright group, and the other half, the oblique group.}

\section{Results and Discussion}

Since the differences between the upright and the oblique groups were minimal $(p>.40)$, we collapsed the results from the two groups. Figure 5B shows the proportion of yes responses as a function of spatial frequency differences between $s_{1}$ and $p_{1}$, measured by the unpredictable onset of $s_{2}$. For comparison, the range of standard errors in Experiment 1's Ignore condition is represented by the gray band, and the Rem 2 condition is represented by an open circle in Figure 5B. The proportion of yes responses for Experiment 3 was essentially the same as the corresponding values from Experiment $1[F(1,7)=0.01, p>.50]$. So, even when the timing of $s_{2}$ 's onset was unpredictable, the subjects still successfully ignored $s_{2}$ and did so in a way that was not different from what we saw with fixed, predictable timing of $s_{2}$. This suggests that ignoring was triggered by such events as the onset of $s_{2}$ and/or the offset of $s_{1}$, not by an exclusively time-based anticipatory process.

Note that the random variation in each trial's ISI and preprobe delay introduced a random delay not only into the timing of $s_{2}$, but also into the timing of $p_{1}$. As a result, from trial to trial, the subjects' memory of $s_{1}$ had to be retained for varying durations. In particular, when added to the other, fixed intervals, random variation in ISI and in preprobe delay combined to produce retention intervals ranging from 1,300 to $4,100 \mathrm{msec}$. To examine the effect of retention interval length, for each subject, the intervals separating $s_{1}$ and $p_{1}$ were sorted into five bins of equal width. Then the proportions correct were calculated for the trials in each bin and are plotted in Figure 6.

We then used least squares to find the best-fitting regression line for each subject's results. A $t$ test showed that the mean slope of these regression lines was not different from zero $[t(7)=0.72, p>.50]$, which confirms that memory for spatial frequency is robust, at least over several seconds (Kahana \& Sekuler, 2002). Moreover, it shows that memory is robust even when an irrelevant stimulus is inserted at a random time into the retention interval.

\section{GENERAL DISCUSSION}

The incomplete but substantial exclusion observed in this study varied with the perceptual difference between the study items and the $p$. Moreover, the results showed that intentional ignoring was triggered not by time-based anticipation of stimulus onset, but by some event, either the onset of the to-be-ignored stimulus or the offset of the item preceding that to-be-ignored stimulus.

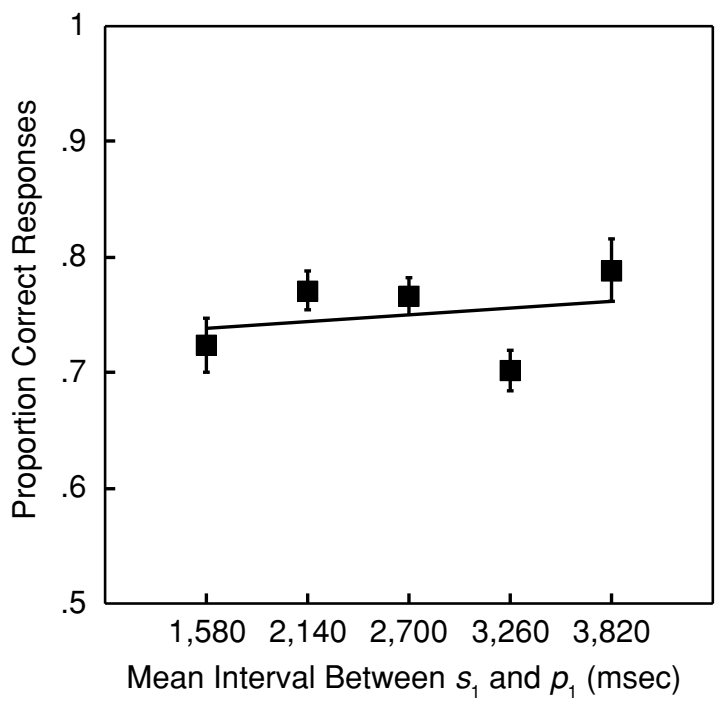

Figure 6. Proportions of correct recognitions as a function of the interval between $s_{1}$ and $p_{1}$, plotted with a linear regression line. Values on the $x$-axis represent the median of each bin. Error bars represent within-subjects standard errors. 


\section{Summed Similarity Framework for Ignoring}

Our results do not unequivocally identify a particular stage or stages of recognition memory - encoding, retention, retrieval, matching, or decision making - at which active control of visual memory operates. For example, the exclusion process might have prevented the stimuli from being encoded, or the behaviorally irrelevant stimulus might have been fully processed in early vision but somehow kept from interfering with the memory of the other study item, during either retention or comparison. To narrow down the stages at which intentional ignoring might possibly have operated, we cast some of our results into the framework of a summed similarity visual recognition memory model. Summed similarity models for visual recognition memory (Kahana \& Sekuler, 2002; Nosofsky, 1986) base yes-no recognition judgments on the summed pairwise similarities between $p$ and each of the study items in turn. When this summed similarity exceeds some criterion value, the model responds yes; the $p$ does match one of the study items. The experiments reported here did not yield sufficient data to support detailed modeling like that in Kahana and Sekuler, but it is worthwhile to examine at least some of our results in a summed similarity framework.

To begin the model-based analysis, we identified trials on which $p_{1}$ and $s_{1}$ were separated by one Weber fraction unit. (Note that in every condition, the study items, $s_{1}$ and $s_{2}$, differed by two Weber fraction units in spatial frequency.) Under these circumstances, as Figure 7 illustrates, $s_{2}$ could be either similar (Case 1) or dissimilar (Case 2) in spatial frequency to $p_{1}$. Because of the differential similarity of $p_{1}$ and $s_{2}$ in the two cases, the summed similarity associated with Case 1 would be larger than the summed similarity with Case 2. In the model framework, this difference in summed similarity means that Case 1 should produce more yes judgments than would Case 2. Of course, if $s_{2}$ had no influence whatever on the summed similarity computation, the resulting judgments should be uninfluenced by $s_{2}$ 's similarity or dissimilarity to $p_{1}$. Because $p_{1}$ actually matched neither $s_{1}$ nor $s_{2}$, these yes judgments consisted of false alarms (erroneous recognitions).
Figure 8 shows the proportion of false alarms (erroneous yes responses) produced in various conditions. The vertical gray bars represent false alarms for Case 1; the dotted bars represent false alarms for Case 2. From left to right, successive pairs of vertical bars are for the Rem2 and Ignore conditions in Experiments 1, 2 and 3, respectively. Note that within each pair of bars, the false alarm rate was higher with $s_{2}$ similar to $p_{1}$ (Case 1) than with $s_{2}$ dissimilar to $p_{1}$ (Case 2), an outcome consistent with the summed similarity framework. The dark gray horizontal bar shows \pm 1 standard error about the mean false alarm rate in the Rem 1 condition. Since $s_{2}$ was absent entirely from the Rem1 condition, this horizontal bar represents the false alarm rate generated exclusively by the presence of $s_{1}$. Because the similarity of both $p_{1}$ and $s_{1}$, as well as $s_{1}$ and $s_{2}$, was controlled in this analysis, differences in false alarm rates in Figure 8 could have come only from differences in similarity of $s_{2}$ and $p_{1}$. Significant deviations upward from the horizontal bar represent significant increases in false alarms, which presumably arose because $s_{2}$ made some significant contribution to summed similarity and, hence, to erroneous recognition.

Consider now the results from the Ignore condition in the three experiments. Comparing the gray vertical bars in Figure 8 shows that when the subjects attempted to ignore $s_{2}$, false alarms occurred at a significantly lower rate than in the Rem 2 condition, when the subjects were attempting to remember both study items ( $p<.001$ for Experiments 1,2, and 3). Furthermore, comparing the rightmost three gray bars against the control value from the Rem1 condition shows that false alarms occurred at a higher rate in the Ignore condition than in the Rem1 condition $(p<$ .02 for Experiments 1 and 3, $p=.06$ for Experiment 2).

This pattern of results shows that when $s_{2}$ was similar to $p_{1}$, the subjects' intention to ignore $s_{2}$ successfully reduced its impact but left a small, significant residual effect. In other words, ignoring appears to have been incomplete. Finally, the dotted bars show that when $s_{2}$ was dissimilar to $p_{1}$, false alarm rates in the Ignore condition did not differ from the false alarm rate in the Rem 1 condi-

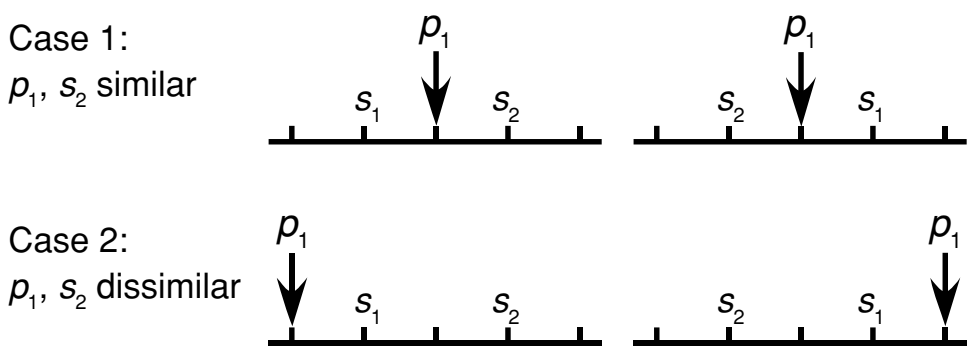

Figure 7. Diagrams illustrating the spatial frequency relationships among $s_{1}$, $s_{2}$, and $p_{1}$ in the Rem 2 and Ignore conditions. In all the cases shown, $s_{1}$ and $p_{1}$ differed by just one Weber unit. The top row illustrates the case in which the spatial frequencies of $s_{2}$ and $p_{1}$ were similar to one another, differing by just one Weber fraction unit. The bottom row illustrates the case in which the spatial frequencies of $s_{2}$ and $p_{1}$ were dissimilar, differing by three Weber fraction units. Within each row, at the left, $s_{1}$ 's frequency is lower than $s_{2}$ 's, and at the right, $s_{1}$ 's frequency is higher than $s_{2}$ 's. 


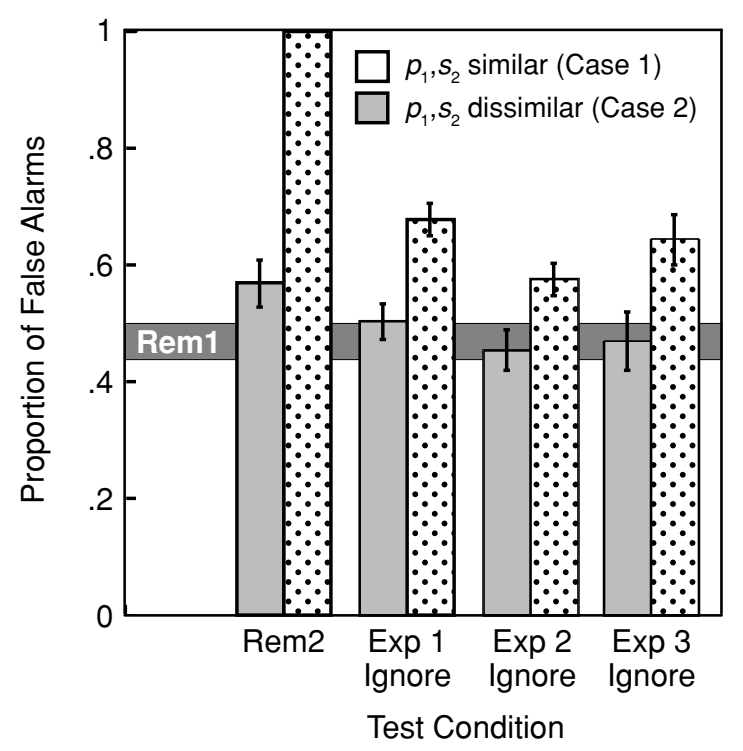

Figure 8. Proportions of false alarms to $p_{1}$ in two conditions in Experiment 1 (Ignore and Rem2) and in Experiments 2 and 3 's Ignore condition. Data are only for trials on which $p_{1}$ differed by one Weber fraction unit from $s_{1}$. Gray bars represent results when $s_{2}$ was perceptually dissimilar to $p_{1}$. Dotted bars represent results when $s_{2}$ was perceptually similar to $p_{1}$. The horizontal bar running across the figure shows mean false alarms, \pm 1 standard error, for $p_{1}$ in the Rem1 condition. Error bars show withinsubjects standard errors. Note that unlike the other figures in the article, poorer performance (more false recognition) is shown as increasing on the vertical axis.

tion ( $p>.45$ for Experiments 1-3). A summed similarity treatment of these conditions suggests that spatial characteristics of $s_{2}$ entered partially into memory even when the subjects were attempting to ignore it, although this effect was small, in comparison with the impact of that same stimulus when $s_{2}$ had to be remembered.

\section{On and Off Gating of Ignoring}

Because the display's mean luminance was held constant throughout each experiment, and because the spatial frequency of the to-be-ignored items varied substantially trial by trial, any event-based intentional ignoring could not have been triggered by low-level features, such as luminance transients or the onset of a particular spatial frequency. And Experiment 2 demonstrated that another low-level feature, orientation, was not the trigger either. In addition, because all the stimuli, behaviorally relevant as well as behaviorally irrelevant, occupied the same region in the visual field, ignoring could not have arisen from some location-dependent attentional process (e.g., Mangun, 1995; Slotnick et al., 2003). Instead, it is likely that higher order processes were involved.

In Experiment 1, we observed both substantial intentional ignoring (the difference between the Ignore and the Rem2 conditions' $p_{1}$ in Figure 4), as well as a small failure of ignoring (the difference between the Rem 1 and the Ignore conditions in Figure 4). We also observed that the characteristics of to-be-ignored stimuli were sufficiently deeply processed to affect the proportion of false alarms (see Figure 8). In addition, Experiment 3 indicated that ignoring is most likely triggered by some event-based process, not by anticipation of the time at which $s_{2}$ would occur. Experiment 3 was not able to distinguish between the possibility that ignoring was triggered by the onset of the to-be-ignored stimulus or by the offset of its preceding stimulus. However, we believe that analysis of false alarms (Figure 8) makes it likely that ignoring was triggered by stimulus onset. That analysis revealed that a taskirrelevant item was at least partially processed, despite the fact that it was supposed to have been ignored. Apparently, before the exclusion process could be fully engaged, the irrelevant stimulus was processed to some degree. In this view, visual registration of the irrelevant stimulus is required for the initiation of its exclusion. As a result, the initial portion of the irrelevant stimulus can enter memory before exclusion takes hold fully. This initial, partial leakage of information into memory could explain why exclusion of the stimulus was only partially successful. Therefore, the failure to achieve complete ignoring might reflect the latency of the exclusion process, rather than any actual imperfection of that process.

Although intentional ignoring might have been gated on, in part, by the onset of the behaviorally irrelevant stimulus, this does not explain how gating might be turned off, as it must be in order for $p$ to be processed. We will offer two speculative hypotheses about the mechanisms that gate off intentional ignoring. The first hypothesis treats termination of intentional ignoring as event based; that is, intentional ignoring is turned off by the end of the irrelevant stimulus (e.g., Seidemann et al., 1998) or by the onset of the next event. An alternative hypothesis characterizes the process as time based. Because $s_{2}$ was always presented for $700 \mathrm{msec}$, it was possible for the subjects to learn the timing, and terminate intentional ignoring when that interval had passed. The present experiments cannot distinguish between these alternatives.

\section{Next Steps}

To maximize the subjects' ability to control memory, the experiments reported here used a block design. As a result, within each block of trials, the subjects could adopt and maintain a consistent strategy. We do not know whether the block design was crucial to success: Would comparable (or any) exclusion be observed if conditions were randomized, with an instructional cue provided just ahead of each trial? And how far in advance would such a cue have to be presented? When trial-specific cues have been used to direct visual attention (e.g., Ball \& Sekuler, 1981), the cues require $\approx 400 \mathrm{msec}$ or more lead time for full effect. If active control of memory operated on a comparably slow time scale, it might be difficult to detect clear signs that memory had been influenced, at least with stimuli presented at or near the rapid timing used here.

Our study has revealed substantial active control over people's recognition of what they have just seen and has 
suggested that this control has its effect after significant visual processing of the to-be-ignored stimulus. Without such control over visual memory for behaviorally irrelevant stimuli, the burden of the processing of visual information under ordinary everyday conditions could substantially subvert memory for items and events that were behaviorally relevant. Such subversion could be especially troubling for many elderly people. As Hasher and Zacks (1988) have shown, some cognitive deficits exhibited by older people arise from diminished inhibitory control over memory (see also Hasher, Tonev, Lustig, \& Zacks, 2001). It would, therefore, be valuable to determine whether older subjects would be disadvantaged in the exclusion paradigm used here.

On the basis of the recognition performance averaged over trials, the intentional ignoring observed in the present study might be described as imperfect. Since this characterization depends on comparisons between groups of trials, the imperfection could have arisen in two distinctly different ways. First, the subjects might have been entirely successful in ignoring the item, but only on a fraction of all trials; on other trials, ignoring would have been ineffective. Alternatively, ignoring could have been fairly consistent over trials, at about the level represented by our mean data. Unfortunately, the present experiments cannot distinguish between these two alternatives, which necessarily limits our understanding of how to-be-ignored items are processed. Further research, such as event-related studies of trial-wise brain activation, could shed significant light on this important question.

\section{REFERENCES}

BALl, K., \& SeKuler, R. (1981). Cues reduce direction uncertainty and enhance motion detection. Perception \& Psychophysics, 30, 119-128.

Bisley, J., Zaksas, D., Droll, J., \& Pasternak, T. (2004). Activity of neurons in cortical area MT during a memory for motion task. Journal of Neurophysiology, 91, 286-300.

Brainard, D. H. (1997). The Psychophysics Toolbox. Spatial Vision, 10, 433-436.

Carrasco, M., Ling, S., \& Read, S. (2004). Attention alters appearance. Nature Neuroscience, 7, 308-313.

Crowder, R. (1978). Mechanisms of auditory backward masking in the stimulus suffix effect. Psychological Review, 85, 502-524.

Hasher, L., Tonev, S., Lustig, C., \& Zacks, R. (2001). Inhibitory control, environmental support, and self-initiated processing in aging. In M. Naveh-Benjamin, M. Moscovitch, \& H. L. Roediger, III (Eds.), Perspectives on human memory and cognitive aging: Essays in honour of Fergus Craik (pp. 286-297). New York: Psychology Press.

HASHER, L., \& ZACKS, R. T. (1988). Working memory, comprehension, and aging: A review and a new view. In G. H. Bower (Ed.), The psychology of learning and motivation (Vol. 22, pp. 193-225). San Diego: Academic Press.

Hole, G. J. (1996). Decay and interference effects in visuospatial shortterm memory. Perception, 25, 53-64.

Hopfinger, J. B., Buonocore, M. H., \& Mangun, G. R. (2000). The neural mechanisms of top-down attentional control. Nature Neuroscience, 3, 284-291.

Hwang, G., Jacobs, J., Geller, A., Danker, J., Sekuler, R., \& KAHANA, M. J. (2005). EEG correlates of verbal and nonverbal working memory. Behavioral \& Brain Functions, 1, 1-13.
Kahana, M. J., \& SeKuler, R. (2002). Recognizing spatial patterns: A noisy exemplar approach. Vision Research, 42, 2177-2192.

Kahana, M. J., Zhou, F., Geller, A., \& Sekuler, R. (in press). Luresimilarity affects visual episodic recognition: Detailed tests of a noisy exemplar model. Memory \& Cognition.

Lalonde, J., \& Chaudhuri, A. (2002). Task-dependent transfer of perceptual to memory representations during delayed spatial frequency discrimination. Vision Research, 42, 1759-1769.

Loftus, G. R., \& Masson, M. E. J. (1994). Using confidence intervals in within-subject designs. Psychonomic Bulletin \& Review, 1, 476490

Luck, S. J., Chelazzi, L., Hillyard, S. A., \& Desimone, R. (1997). Neural mechanisms of spatial selective attention in areas V1, V2, and V4 of macaque visual cortex. Journal of Neurophysiology, 77, 24-42.

MAcLeod, C. M. (1998). Directed forgetting. In J. M. Golding \& C. M. MacLeod (Eds.), Intentional forgetting: Interdisciplinary approaches (pp. 1-57). Mahwah, NJ: Erlbaum.

Macrae, C. N., Bodenhausen, G. V., Milne, A. B., \& Ford, R. L. (1997). On the regulation of recollection: The intentional forgetting of stereotypical memories. Journal of Personality \& Social Psychology, 72, 709-719.

Magnussen, S., Greenlee, M. W., Asplund, R., \& Dyrnes, S. (1991). Stimulus-specific mechanisms of visual short-term memory. Vision Research, 31, 1213-1219.

Magnussen, S., Greenlee, M. W., \& Thomas, J. P. (1996). Parallel processing in visual short-term memory. Journal of Experimental Psychology: Human Perception \& Performance, 22, 202-212.

Mangun, G. R. (1995). Neural mechanisms of visual selective attention. Psychophysiology, 32, 4-18.

Nosofsky, R. M. (1986). Attention, similarity, and the identification categorization relationship. Journal of Experimental Psychology: General, 115, 39-57.

Ostendorf, F., Finke, C., \& Ploner, C. (2004). Inhibition of visual discrimination during a memory-guided saccade task. Journal of Neurophysiology, 92, 660-664.

Parmentier, F. B. R., Tremblay, S., \& Jones, D. M. (2004). Exploring the suffix effect in serial visuospatial short-term memory. Psychonomic Bulletin \& Review, 11, 289-295.

Pelli, D. G. (1997). The VideoToolbox software for visual psychophysics: Transforming numbers into movies. Spatial Vision, 10, 437-442.

Pelli, D. G., Robson, J. G., \& Wilkins, A. J. (1988). Designing a new letter chart for measuring contrast sensitivity. Clinical Vision Sciences, 2, 187-199.

Seidemann, E., Zohary, E., \& Newsome, W. (1998). Temporal gating of neural signals during performance of a visual discrimination task. Nature, 394, 72-75.

Sekuler, R., Kahana, M. J., Mclaughlin, C., Golomb, J., \& WingField, A. (2005). Preservation of episodic visual recognition memory in aging. Experimental Aging Research, 31, 1-13.

Slotnick, S. D., Schwarzbach, J., \& Yantis, S. (2003). Attentional inhibition of visual processing in human striate and extrastriate cortex. Neurolmage, 19, 1602-1611.

Somers, D. C., Dale, A. M., Seiffert, A. E., \& Tootell, R. B. (1999). Functional MRI reveals spatially specific attentional modulation in human primary visual cortex. Proceedings of the National Academy of Sciences, 96, 1663-1668.

StERnBerg, S. (1966). High-speed scanning in human memory. Science, 153, 652-654.

STERNBERG, S. (1975). Memory scanning: New findings and current controversies. Quarterly Journal of Experimental Psychology, 27, 1-32.

Toms, M., Morris, N., \& Foley, P. (1994). Characteristics of visual interference with visuospatial working memory. British Journal of Psychology, 85, 131-144.

TREUE, S. (2001). Neural correlates of attention in primate visual cortex. Trends in Neurosciences, 24, 295-300.

WASHBURn, D. A., \& Astur, R. S. (1998). Nonverbal working memory of humans and monkeys: Rehearsal in the sketchpad? Memory \& Cognition, 26, 277-286.

Watanabe, T., Harner, A., Miyauchi, S., Sasaki, Y., Nielsen, M., 
Palomo, D., \& MukaI, I. (1998). Task-dependent influences of attention on the activation of human primary visual cortex. Proceedings of the National Academy of Sciences, 95, 11489-11492.

WEGNER, D. M. (1994). Ironic processes of mental control. Psychological Review, 101, 34-52.

WenZlafF, R. M., \& Wegner, D. M. (2000). Thought suppression. Annual Review of Psychology, 51, 59-91.
Zhou, F., Kahana, M. J., \& Sekuler, R. (2004). Short-term episodic memory for visual textures: A roving probe gathers some memory. Psychological Science, 153, 112-118.

(Manuscript received October 22, 2004; revision accepted for publication May 18, 2005.) 